\title{
Special issue "Characterization of the geomagnetic field and its dynamic environment using data from space-based magnetometers"
}

\author{
C. Stolle ${ }^{1,2^{*}}$, N. Olsen ${ }^{3}$, B. Anderson ${ }^{4}$, E. Doornbos ${ }^{5}$ and A. Kuvshinov ${ }^{6}$
}

The main part of the geomagnetic field arises from electric currents in the Earth's outer core. It extends to a distance of $\sim 10$ Earth radii and acts as a shield for protecting our atmosphere against solar and cosmic particle radiation. It also determines the strength and geometry of ionospheric and magnetospheric current systems. While the Earth's core field varies on time scales of months to years, electric currents in the ionosphere and magnetosphere change within seconds to days, e.g., during space weather events. Continuous monitoring of the various magnetic field variations is thus important to characterize the Earth's space environment and ensure the preparedness of modern technology on the ground and in space on which society increasingly depends.

Most of our knowledge of the spatial and temporal variations of the recent geomagnetic field has been obtained using observations taken by high precision magnetic satellite missions, such as Ørsted, CHAMP, and currently the European Space Agency's (ESA) magnetic field constellation mission Swarm. However, a multitude of satellites in low Earth orbit (LEO) carry magnetometers that, by design, do not meet the accuracy of dedicated geomagnetic missions. These are avionic, so-called platform magnetometers that are primarily used for coarse attitude determination of the spacecraft. Another category is magnetometers meant for science applications mounted

\footnotetext{
${ }^{*}$ Correspondence: cstolle@gfz-potsdam.de

${ }^{1}$ Helmholtz Centre Potsdam, GFZ German Research Centre

for Geosciences, Telegrafenberg, 14473 Potsdam, Germany

Full list of author information is available at the end of the article
}

on a boom, but those missions often neither measure the absolute magnetic field intensity together with the variations of the magnetic components nor provide precise attitude determination of the magnetometer itself. However, magnetic data from these missions have been shown to add valuable information in characterizing the geomagnetic field and its environment after appropriate calibration.

This special issue collects articles that document successful calibration strategies including the validation of their results, e.g., by comparison to other data or applied in exemplary science cases, and articles that outline valuable research application in Earth and space sciences by the use of these magnetometer data. Figure 1 shows the altitude distribution of the satellite missions, which data were analyzed in papers of this special issue. In combination, they provide a multi-mission data set in LEO spanning almost two solar cycles.

Extending the calibration scheme developed and applied to data from high-precision satellite missions, Olsen et al. (2020) introduce an approach for characterization, calibration, and alignment of vector magnetometer data, applied to data from the platform magnetometers onboard ESA's CryoSat- 2 satellite. The calibration is performed by comparing the magnetometer sensor readings with magnetic field values for the time and position of the satellite as given by an a priori high-precision geomagnetic field model, e.g. CHAOS (Finlay et al. 2020), to estimate the magnetometer calibration parameters by solving a least square problem. The calibrated magnetic data show good agreement with 


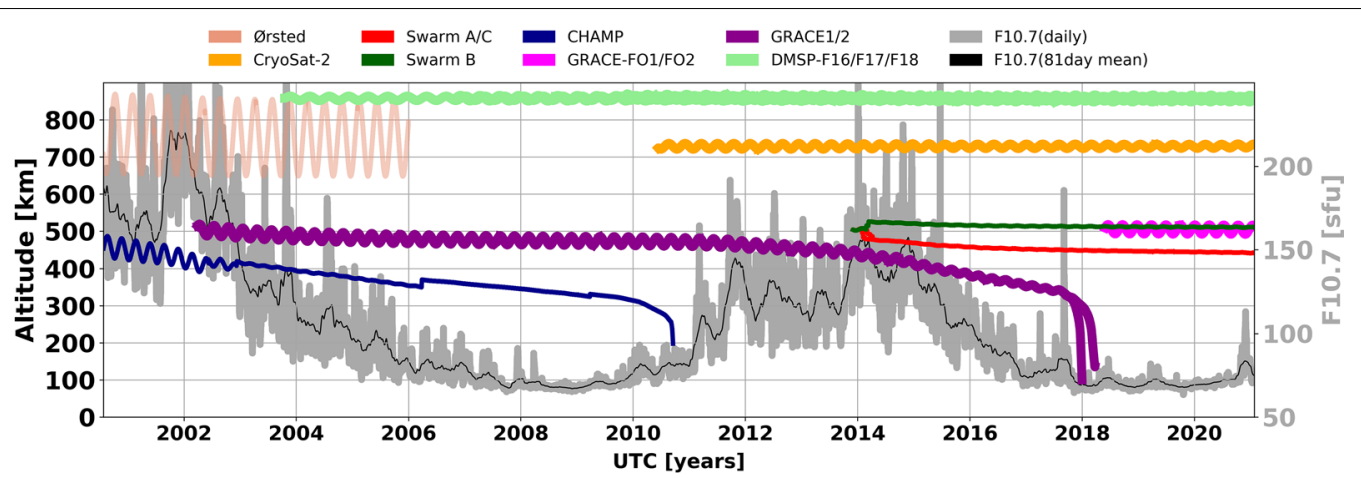

Fig. 1 Altitude of satellite missions used in this special issue (left scale) and solar flux index $F_{10.7}$ (right scale). Altitude of high-precision magnetic missions are plotted by thinner lines, those of others by bolder lines. Altitudes between 26 July 2000 and 24 January 2021 are shown. Ørsted altitudes are shown up to 12/2005 until the mission provided high-precision magnetic data

Swarm satellite magnetic measurements during close encounters. Furthermore, it was demonstrated that the CryoSat-2 magnetic variations follow well the Dst-index during a geomagnetic storm event.

Stolle et al. (2021) applied a similar least-square fit to platform magnetometer data of the GRACE-FO dualsatellite constellation mission. The average mean of the remaining residuals to the CHAOS model used in the calibration process show standard deviations below $10 \mathrm{nT}$ rms, which are similar to those derived for CryoSat-2. By combining the Swarm, GRACE-FO, and CryoSat-2 satellites, the authors showed that the extended "constellation" does well capture the local time evolution of the magnetospheric ring current under geomagnetic storm conditions. Also, GRACE-FO-derived auroral field-aligned currents compare well in amplitude with those derived from Swarm data. The pearls-on-a-string configuration of the GRACE-FO mission further allows scale analyses of ionospheric structures.

Olsen (2021) extended the scheme to process and calibrate the platform magnetometer data of the GRACE dual-satellite mission. The remaining residuals to the models are somewhat higher than those for CryoSat-2 and GRACE-FO due to lacking direct measurements of magnetometer temperature and the rather coarse 12-bit discretization of the GRACE magnetometers; however, the data have been proven relevant for geophysical applications in publications mentioned below. Besides, by combining data from the Swarm, GRACE, and CryoSat-2 satellites in spherical harmonic ( $\mathrm{SH}$ ) analyses, the author confirms that the local time asymmetric part of the magnetospheric ring current after the peak of a geomagnetic storm decays faster compared to its symmetric part.

Alken et al. (2020) provide the Frontier Letter for this special issue. They describe a novel scheme for co-estimating magnetometer calibration parameters together with a model of Earth's magnetic field. This method does not require an a priori geomagnetic field model such as CHAOS but relies on supporting calibrated magnetic data, here provided by CHAMP, Swarm, and ground observatories. The authors apply this scheme to calibrate magnetic data from the DMSP and CryoSat- 2 missions to enhance the description of the rapid core field evolution when satellite-based high-precision magnetic data were not available, e.g., during the gap period between CHAMP and Swarm in 2010-2013 (see Fig. 1).

Kloss et al. (2021) present a similar approach, go further, and co-estimate both the internal (core) and the external (magnetospheric) part of geomagnetic field models along with magnetometer calibration parameters. By that, they derive a geomagnetic field model spanning 2008 to 2018 with satellite magnetic data from CHAMP, Swarm, data from ground observatories, and platform magnetometer data from CryoSat-2 and the GRACE satellite pair. It was proven that platform magnetometer data provide additional information on the secular acceleration, especially in the Pacific Ocean region during the gap between CHAMP and Swarm.

Hammer et al. (2021) use time series of geomagnetic secular variation at 300 globally distributed geomagnetic virtual observatories to study time variations of the Earth's core magnetic field both at satellite altitude and at the core-mantle boundary. They use 20 years of continuous magnetic field measurements from the Ørsted, CHAMP, and Swarm satellite missions and calibrated platform magnetometer data from the CryoSat-2 satellite. The authors find that CryoSat- 2 platform magnetometer data provide a valuable contribution to emerging pictures of regional sub-decadal core field variations.

Velímský and Knopp (2021) and Kuvshinov et al. (2021) use up to 6 years of contemporaneous magnetic observations of Swarm and CryoSat-2 to determine the 
three-dimensional (3-D) structure of mantle conductivity. The largest benefit from combining the missions was through the enhanced coverage of local time sectors at same observational periods. In their works, the teams followed different approaches.

Velímský and Knopp (2021) perform time-domain forward and inverse electromagnetic (EM) induction modeling to the latest version of satellite-derived time series of SH coefficients of external (inducing) and internal (induced) parts of the magnetic potential describing signals of magnetospheric origin. The inclusion of CryoSat-2 platform magnetometer data had only small influence on the inversion results, but allow for a larger reduction of the data misfit in the applied inversion. The authors recovered large-scale conductivity structures (parameterized by SH up to degree 3) in Earth's mantle, which partially overlap with the shape of the large lowshear velocity provinces in the lower mantle.

Kuvshinov et al. (2021) rely on the estimation and inversion of the so-called matrix $Q$-responses, which relate in the frequency domain the $\mathrm{SH}$ coefficients of inducing and induced parts of the magnetic potential. Their results show a significant deviation of mantle conductivity distribution from the global 1-D conductivity profile in the Pacific Ocean region. In their outlook, the authors emphasized the need for the data from more and better distributed (in terms of local time coverage) satellite missions; this will allow for an improved description of the source geometry in the ionosphere and magnetosphere, the prerequisite for future advances in global 3-D EM mapping of mantle conductivity.

Looking at studies of ionospheric current systems, Xiong et al. (2020) compare auroral field-aligned currents (FAC) derived from magnetic data of the DMSP mission with simultaneous energetic particle fluxes detected at the same spacecraft. They performed the first statistical study of these parameters' relation from simultaneous data and found systematic differences in the location of peaks of particle energy flux and large-scale FACs, emphasizing the complexity of the auroral oval region. Furthermore, during conjunction events, the DMSP field-aligned currents were proven to be similar in amplitude with Swarm-derived results further supporting their value for space science.

Park et al. (2020) demonstrate that calibrated platform magnetic data can also be used in statistical investigations of low amplitude ionospheric currents at non-polar latitudes. From vertical currents detected during 8 years at CryoSat- 2 and during nearly 2 years at GRACE-FO the authors successfully derive global pattern of interhemispheric FACs, the distribution of which is dominated by seasonal variations and tidal forcing from the lower atmosphere. They further discussed that the direction of the currents is systematic southward throughout the year in the South Atlantic sector due to the predominant geometry and relatively low strength of the magnetic field in that region. The paper provides observational evidences of noontime dynamo currents at the highest altitude (of about $700 \mathrm{~km}$, sampled by CryoSat-2) reported so far.

In conclusion, appropriately calibrated data from nondedicated magnetometers onboard LEO satellites have high value for both Earth's and space research. Examples include (1) filling periods of unavailability of high-precision data, (2) the combination of several missions to a "constellation" for better spatio-temporal coverage, and (3) the availability of geomagnetic observations at multiple platforms, which provides an extended opportunity to perform combined analysis with other ionospheric parameters sampled by particular missions. It is nonetheless noteworthy that these magnetic data have been calibrated along with high-precision observations or using high-resolution geomagnetic field models derived from measurements by the Swarm satellites. Simultaneous data from at least one dedicated high-precision magnetic satellite mission thus remain crucial for this endeavor.

\section{Abbreviations \\ CHAMP: CHAllenging Minisatellite Payload; CHAOS: CHAmp Ørsted SAC-C magnetic field model; DMSP: Defense Meteorological Satellite Program; Dst: Geomagnetic Equatorial Disturbance Storm Time Index; EM: Electromagnetic; ESA: European Space Agency; FAC: Field-Aligned Currents; GRACE(-FO): Gravity Recovery and Climate Experiment (-Follow-On); LEO: Low Earth Orbit; rms: Root Mean Square; SH: Spherical Harmonic; 1-D: One-dimensional; 3-D: Three-dimensional.}

\section{Acknowledgements}

We express our sincere gratitude to the authors who contributed to this special issue and the reviewers who evaluated the contributions and gave thoughtful comments and suggestions. We also thank Editors-in-Chief Yasuo Ogawa and Takeshi Sagiya for their support and the various institutions and entities that operate satellites and provide data.

\section{Authors' contributions}

All authors of this article served as guest editors for this special issue. All authors read and approved the final manuscript.

\section{Funding}

This initiative has been partly supported by Swarm DISC activities funded by ESA under contract no. 4000109587/13//-NB.

\section{Declarations}

Competing interests

The authors declare that they have no competing interests.

\section{Author details}

${ }^{1}$ Helmholtz Centre Potsdam, GFZ German Research Centre for Geosciences, Telegrafenberg, 14473 Potsdam, Germany. ${ }^{2}$ Faculty of Science, University of Potsdam, Karl-Liebknecht-Str. 24-25, 14476 Potsdam, Germany. ${ }^{3}$ Division of Geomagnetism and Geospace, DTU Space, Technical University of Denmark, Centrifugevej 356, 2800 Kongens Lyngby, Denmark. ${ }^{4}$ Applied Physics Laboratory, Johns Hopkins University, 11100 Johns Hopkins Rd, Laurel, MD, USA. ${ }^{5}$ Royal Netherlands Meteorological Institute (KNMI), Utrechtseweg 297, 
3731 GA De Bilt, The Netherlands. ${ }^{6}$ Institute of Geophysics, ETH Zürich, Sonneggstrasse, 8092 Zurich, Switzerland.

Received: 25 March 2021 Accepted: 26 March 2021

Published online: 02 April 2021

\section{References}

Alken P, Olsen N, Finlay C (2020) Co-estimation of geomagnetic field and inorbit fluxgate magnetometer calibration parameters. Earth Planets Space 72. https://doi.org/10.1186/s40623-020-01163-9

Finlay C, Kloss C, Olsen N, M H, Tøffner-Clausen L, Grayver A, Kuvshinov A (2020) The CHAOS-7 geomagnetic field model and observed changes in the South Atlantic Anomaly. Earth Planets Space 72. https://doi.org/10. 1186/s40623-020-01252-9

Hammer MD, Finlay CC, Olsen N (2021) Applications of CryoSat-2 satellite magnetic data in studies of the Earth's core field variations. Earth Planets Space 73. https://doi.org/10.1186/s40623-021-01365-9

Kloss C, Finlay CC, Olsen N (2021) Co-estimating geomagnetic field and calibration parameters: modeling Earth's magnetic field with platform magnetometer data. Earth Planets Space 73. https://doi.org/10.1186/ s40623-020-01351-7

Kuvshinov A, Grayver A, Tøffner-Clausen L, Olsen N (2021) Probing 3-D electrical conductivity of the mantle using 6 years of Swarm, CryoSat- 2 and observatory magnetic data and exploiting matrix Q-responses approach. Earth Planets Space 73. https://doi.org/10.1186/s40623-020-01341-9
Olsen N (2021) Magnetometer data from the GRACE satellite duo. Earth Planets Space 73. https://doi.org/10.1186/s40623-021-01373-9

Olsen N, Albini G, Bouffard J, Parrinello T, Tøffner-Clausen L (2020) Magnetic observations from CryoSat-2: calibration and processing of satellite platform magnetometer data. Earth Planets Space 72:48. https://doi.org/ 10.1186/s40623-020-01171-9.

Park J, Stolle C, Yamazaki Y, Rauberg J, Michaelis I, Olsen N (2020) Diagnosing low-/mid-latitude ionospheric currents using platform magnetometers: CryoSat-2 and GRACE-FO. Earth Planets Space 72. https://doi.org/10. 1186/s40623-020-01274-3

Stolle C, Michaelis I, Xiong C, Rother M, Usbeck T, Yamazaki Y, Rauberg J, Styp-Rekowski K (2021) Observing Earth's magnetic environment with the GRACE-FO mission. Earth Planets Space 73. https://doi.org/10.1186/ s40623-021-01364-w

Velímský J, Knopp O (2021) Lateral variations of electrical conductivity in the lower mantle constrained by Swarm and CryoSat-2 missions. Earth Planets Space 73. https://doi.org/10.1186/s40623-020-01334-8

Xiong C, Stolle C, Alken P, Rauberg J (2020) Relationship between large-scale ionospheric field-aligned currents and electron/ion precipitations: DMSP observations. Earth Planets Space 72. https://doi.org/10.1186/ s40623-020-01286-Z

\section{Publisher's Note}

Springer Nature remains neutral with regard to jurisdictional claims in published maps and institutional affiliations.

\section{Submit your manuscript to a SpringerOpen ${ }^{\odot}$ journal and benefit from:}

- Convenient online submission

- Rigorous peer review

- Open access: articles freely available online

- High visibility within the field

- Retaining the copyright to your article

Submit your next manuscript at $\boldsymbol{\Delta}$ springeropen.com 